\title{
Colonoscopic Instillation of Coca-Cola for Evacuation of Large Fecaloma: A Report of Two Cases and Review of Literature
}

\author{
Avnish Kumar Seth ${ }^{1, \odot ~ M a h e s h ~ K u m a r ~ G u p t a ~}{ }^{1} \quad$ Rinkesh Kumar Bansal ${ }^{1} \quad$ Radha Krishan Verma ${ }^{2}$ \\ Gursimran Kaur ${ }^{1}$ \\ ${ }^{1}$ Department of Gastroenterology \& Hepatobiliary Sciences, Fortis \\ Memorial Research Institute, Gurugram, Haryana, India \\ ${ }^{2}$ Department of Radiology, Fortis Memorial Research Institute, \\ Gurugram, Haryana, India \\ Address for correspondence Avnish Kumar Seth, MBBS, MD, DM, \\ Department of Gastroenterology \& Hepatobiliary Sciences, Fortis \\ Memorial Research Institute, Sector 44, Gurugram 122002, Haryana, \\ India (e-mail: akseth2003@yahoo.com).
}

J Health Allied Sci ${ }^{\mathrm{NU}}$ 2022;12:98-100.

\begin{abstract}
Introduction Fecaloma is a large mass of organized hardened feces causing impaction, usually in rectum and sigmoid colon. Medical management usually entails digital evacuation, use of clearance enema, and oral laxatives. We report two patients managed successfully with colonoscopic instillation of Coca-Cola and review the literature. Case Report Patient 1: A 37-year-old male presented with firm, nontender, pitting mass over lower abdomen for 2 months and inability to pass stool for 1 month. Per-rectal examination and imaging confirmed presence of solid stool with gross distension of rectum and sigmoid colon. Attempts at clearance of stool with conventional methods were unsuccessful. At colonoscopy, $4 \mathrm{~L}$ of Coca-Cola was instilled into descending and sigmoid colon, leading to evacuation of $10 \mathrm{~L}$ of fragmented and liquid stool.

Patient 2: A 72-year-old diabetic lady presented with constipation and tender, firm pelvic mass extending till mid-abdomen for 6 months. Per-rectal examination revealed presence of hard stool. Imaging confirmed large amount of fecal matter in dilated rectum, sigmoid, and descending colon. Attempts at evacuating stool with digital evac-

- Keywords

- fecaloma

- colonoscopic removal

- colonoscopic installation

- Coca-Cola uation, sodium phosphate enema, and oral polyethylene glycol were unsuccessful. At colonoscopy, two sittings of instillation of $990 \mathrm{~mL}$ of Coca-Cola Light each were done into sigmoid colon over 2 days, resulting in clearance.

Conclusion Colonoscopic instillation of Coca-Cola may be effective in evacuation of large fecaloma from rectum, sigmoid, and descending colon when refractory to use of conventional methods like digital disimpaction, rectal enema, and oral laxatives.
\end{abstract}

\section{Introduction}

Fecaloma, first described in 1967, is a large mass of organized hardened feces causing impaction, mostly in rectum and sigmoid colon. ${ }^{1}$ Most fecalomas may be successfully removed by treatment with digital evacuation and use of bowel cleansing enema and oral laxatives. Surgical intervention is rarely required. We report two patients in whom large fecalomas published online August 5, 2021
DOI https://doi.org/

$10.1055 / \mathrm{s}-0041-1734340$

ISSN 2582-4287

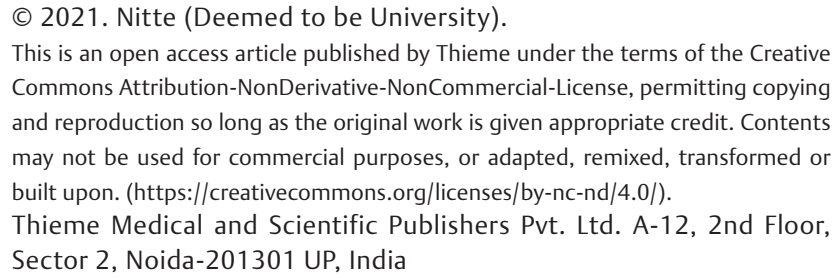
and reproduction so long as the original work is given appropriate credit. Contents may not be used for commercial purposes, or adapted, remixed, transformed or built upon. (https://creativecommons.org/licenses/by-nc-nd/4.0/). Thieme Medical and Scientific Publishers Pvt. Ltd. A-12, 2nd Floor, Sector 2, Noida-201301 UP, India 
were successfully evacuated with colonoscopic instillation of Coca-Cola.

\section{Patient 1}

A 37-year-old male, with constipation since early childhood, presented with mass over lower abdomen for 2 months and inability to pass stool for 1 month. There was no history of cessation of flatus, hematochezia, fever, weight loss, or urinary symptoms. He had not been evaluated for constipation earlier and was using laxatives in various combinations including ispaghula, prucalopride, senna, castor oil, bisacodyl, and lactulose since childhood. On examination, there was firm, nontender, pitting mass in the pelvis and abdomen with upper border close to umbilicus. The mass was dull to percussion. Bowel sounds were normal. Hard impacted stool was felt on per-rectal examination. X-ray of abdomen showed gross distension of rectum and sigmoid colon with copious

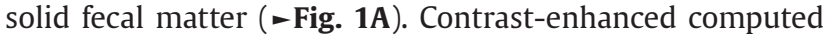
tomography (CT) abdomen revealed no bowel thickening, ascites, or lymph node enlargement. Attempts at clearance of stool with digital fragmentation, sodium phosphate enema, and oral polyethylene glycol were unsuccessful. At limited colonoscopy (CF-H180AL, manufactured by Olympus Medical Systems Corp at Tokyo, Japan), large clumps of solid fecal matter were noted in rectum, sigmoid, and descending colon. Following informed consent, $4 \mathrm{~L}$ of Coca-Cola was instilled with $50 \mathrm{~mL}$ syringe through the working channel of colonoscope into descending and sigmoid colon (-Fig. 1B). The patient was asked to change position to supine, right lateral, prone and left lateral every 5 minutes. Over the next 1 hour, he evacuated over $10 \mathrm{~L}$ of fragmented and liquid foul-smelling stool in a bed-side commode. Repeat X-ray abdomen showed marked clearance of stool with a transition zone between distal rectum and the dilated colon suggestive of Hirschsprung's disease (-Fig. 1C). The patient did not give consent for anorectal manometry or full-thickness rectal biopsy and was discharged on laxatives. He remains lost to follow-up.

\section{Patient 2}

A 72-year-old lady, well controlled on treatment for diabetes mellitus and hypothyroidism, presented with constipation

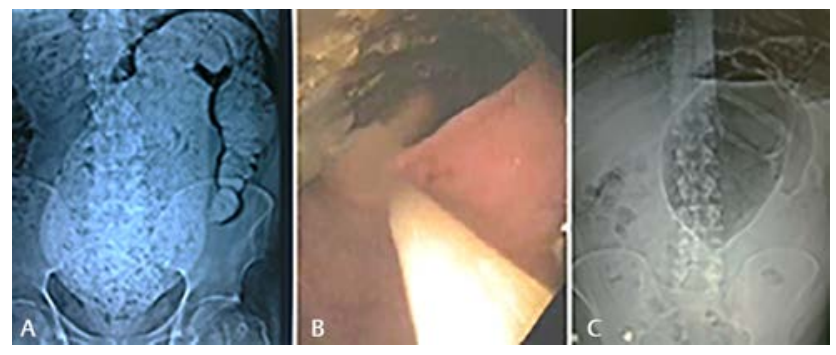

Fig. 1 (A) Plain skiagram of abdomen showing gross distension of rectum and sigmoid colon with solid fecal matter. (B) Instillation of Coca-Cola through working channel of colonoscope in descending and sigmoid colon. (C) Plain skiagram showing complete evacuation of stool from rectum and sigmoid colon with possible transition zone in rectum. and progressive distension of abdomen for 6 months. She was able to pass only small amount of mucus. There was no history of cessation of flatus, hematochezia, pain abdomen, fever, or weight loss. She had not been evaluated for constipation and was using laxatives like ispaghula, prucalopride, and polyethylene glycol. Physical examination revealed ill-defined, firm, tender mass in pelvis and extending to the hypogastrium, left iliac, umbilical, and left lumbar regions. The mass was tympanic on percussion and bowel sounds were normal. Per-rectal examination revealed normal anal tone and presence of hard stool. X-ray abdomen revealed large amount of fecal matter in dilated rectum, sigmoid, and descending colon with dilatation of proximal colon. CT scan of abdomen showed fecal loading with grossly distended rectum, sigmoid colon (diameter $12.7 \mathrm{~cm}$ ) and descending colon with displacement of small bowel, mesenteric vessels, left ureter and urinary bladder ( - Fig. 2A). Repeated attempts at evacuating stool with digital evacuation, sodium phosphate enema, and oral polyethylene glycol were unsuccessful. Colonoscopy, performed with low air pressure, revealed grossly dilated rectum and sigmoid colon with large amount of solid fecal matter ( $\mathbf{- F i g . 2 B}$ ). The patient refused the option of surgical treatment. Following high risk informed consent, three cans of Coca-Cola Light, total of $990 \mathrm{~mL}$, were instilled into sigmoid colon through the working channel of colonoscope. Within 1 hour she passed explosive stool and expelled $2 \mathrm{~L}$ of fecal matter. As clinically and radiologically there was partial clearance of fecaloma, the procedure was repeated the following day. The abdominal distension decreased and CT abdomen showed a remarkable decrease in the fecal loading ( - Fig. 2B). Patient was discharged on request the following day on prucalopride and polyethylene glycol. On telephonic follow-up, her mega-colon did not subside and she required emergency surgery for perforation of sigmoid colon 3 months later. She remains well on ileostomy.

\section{Discussion}

Fecaloma, a large mass of impacted stool resulting in deformity of the intestine, is usually located in rectum (70\%), sigmoid colon (20\%), and rarely (10\%) in the rest of colon. ${ }^{1}$ The propensity for occurrence in distal colon is ascribed to smaller lumen, hardness of stool, and poor vascular supply along
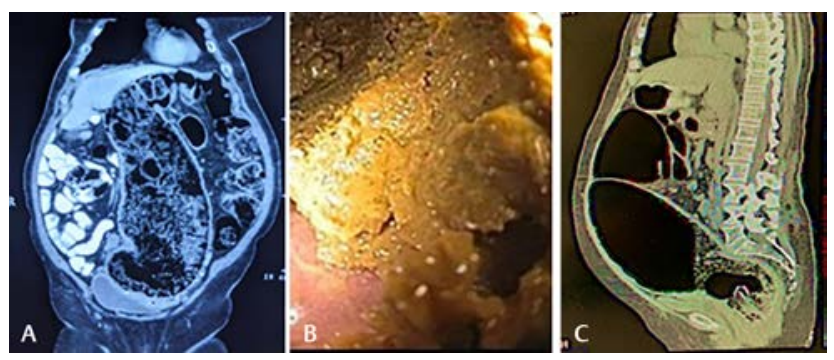

Fig. 2 (A) Coronal view of computed tomography (CT) scan of abdomen showing fecal matter in grossly distended rectum, sigmoid, and descending colon. (B) Colonoscopy showing solid fecal matter in sigmoid colon. (C) Sagittal view of CT scan showing complete clearance of rectum and sigmoid colon. 
antimesenteric border. ${ }^{2}$ Ileal fecaloma causing intestinal obstruction in a child has been reported. ${ }^{3}$ Common causes of fecaloma include chronic constipation, especially in elderly, spinal injury, and neuropsychiatric disorders. Patients with Hirschsprung's disease, Chagas disease, and colonic obstruction due to tumors, tuberculosis, or Crohn's disease may also present with fecaloma. Clinical manifestations include mass in pelvis and lower abdomen, pain, paradoxical or overflow diarrhea, hematochezia due to stercoral ulceration, urinary retention, or even colonic perforation. ${ }^{4}$ Fecaloma must be differentiated from toxic megacolon due to acute colitis. Conservative treatment described includes digital fragmentation, cleansing enema, and oral polyethylene glycol. Rarely, surgery may be indicated when fecaloma is refractory to medical treatment or there are signs of peritonitis. Sakai et al described endoscopic removal of $5 \mathrm{~cm}$ fecaloma in a 78-year-old patient. ${ }^{5}$ There are few reports of fecaloma from India, managed both conservatively and with surgery. ${ }^{6,7}$

The use of Coca-Cola for the treatment of gastric phytobezoars and clearing blocked enteral feeding tubes is well established. ${ }^{8,9}$ The dissolution power of Coca-Cola is a result of $\mathrm{pH}$ of 2.6 due to carbonic and phosphoric acid, mucolytic effect of sodium bicarbonate, and carbon dioxide bubbles. ${ }^{10}$ There are only two previous reports of use of Coca-Cola for management of fecaloma. Lee and Kim injected Coca-Cola into $4.7 \mathrm{~cm}$ rectal fecaloma with sclerosing needle. ${ }^{11}$ Ontanilla Clavijo et al reported use of combination of injection and colonic wash with $500 \mathrm{~mL}$ of Coca-Cola followed by fragmentation with snare. ${ }^{12}$ Both our patients had extensive fecalomas, not amenable to injection of Coca-Cola with sclerosing needle. We also used higher volume of $4 \mathrm{~L}$ for the colonic wash in patient 1 and two aliquots of about a liter each for patient 2 . Though all reports in literature report use of Coca-Cola, any cola should be effective. Diet Coke, Coca-Cola Light or Zero, containing aspartame as sweetener, may be more suitable for use in diabetics, and are reported to be as effective as regular Coca-Cola for dissolution of gastric phytobezoars. ${ }^{13}$ Although no complication of use of Coca-Cola has been described in a systematic review on use in gastric phytobezoars, the thinned out wall of distal megacolon may be prone to perforation as a result of barotrauma due to air insufflation at colonoscopy and release of carbon dioxide bubbles. Air pressure setting should be kept at 'low' and no attempt should be made to complete a full colonoscopy. Coca-Cola should be used with caution and in smaller aliquots, like in patient 2 , in the presence of tenderness over distended sigmoid colon and presence of tympanic percussion note. Contraindications to the use of Coca-Cola would include presence of fever, abdominal guarding, absence of bowel sounds, and presence of gas under diaphragm. Recurrence of fecaloma is common and patients should be maintained on prucalopride and laxatives till definitive treatment of the cause, where possible. Use of oral lactulose should be avoided as it increases gaseous distension. Mortality rate may be as high as $33 \%$ in hospitalized patients with fecaloma, especially in the presence of colonic perforation, advance age, and comorbidities. ${ }^{4}$

\section{Conclusion}

Colonoscopic instillation of Coca-Cola may be effective in evacuation of large fecaloma from rectum, sigmoid, and descending colon when refractory to use of conventional methods like digital disimpaction, rectal enema, and oral laxatives.

\section{Conflict of Interest}

None declared.

\section{References}

1 Abella ME, Fernández AT. Large fecalomas. Dis Colon Rectum 1967;10(5):401-404

2 Serrano Falcón B, Barceló López M, Mateos Muñoz B, Álvarez Sánchez A, Rey E. Fecal impaction: a systematic review of its medical complications. BMC Geriatr 2016;16:4

3 Yoo HY, Park HW, Chang SH, Bae SH. Ileal fecaloma presenting with small bowel obstruction. Pediatr Gastroenterol Hepatol Nutr 2015;18(3):193-196

4 Park JS, Park TJ, Hwa JS, Seo JH, Park CH, Youn HS. Acute urinary retention in a 47-month-old girl caused by the giant fecaloma. Pediatr Gastroenterol Hepatol Nutr 2013;16(3):200-205

5 Sakai E, Inokuchi $\mathrm{Y}$, Inamori $\mathrm{M}$, et al. Rectal fecaloma: successful treatment using endoscopic removal. Digestion 2007;75(4):19810.1159/000108627

6 Rajagopal A, Martin J. Giant fecaloma with idiopathic sigmoid megacolon: report of a case and review of the literature. Dis Colon Rectum 2002;45(6):833-835

7 Aiyappan SK, Ranga U, Samraj A, Rajan SC, Veeraiyan S. A case of fecaloma. Indian J Surg 2013;75(4):323-324

8 Iwamuro M, Okada H, Matsueda K, et al. Review of the diagnosis and management of gastrointestinal bezoars. World J Gastrointest Endosc 2015;7(4):336-345

9 Dandeles LM, Lodolce AE. Efficacy of agents to prevent and treat enteral feeding tube clogs. Ann Pharmacother 2011;45(5):676-680

10 Ladas SD, Kamberoglou D, Karamanolis G, Vlachogiannakos J, Zouboulis-Vafiadis I. Systematic review: Coca-Cola can effectively dissolve gastric phytobezoars as a first-line treatment. Aliment Pharmacol Ther 2013;37(2):169-173

11 Lee JJ, Kim JW. Successful removal of hard sigmoid fecaloma using endoscopic cola injection. Korean J Gastroenterol 2015;66(1):46-49

12 Ontanilla Clavijo G, León Montañés R, Sánchez Torrijos Y, López Ruiz T, Bozada García JM. Colonic obstruction secondary to sigmoid fecaloma endoscopically resolved with Coca-Cola ${ }^{\circledR}$. Rev Esp Enferm Dig 2017;109(4):306-308

13 Ladas SD, Triantafyllou K, Tzathas C, Tassios P, Rokkas T, Raptis SA. Gastric phytobezoars may be treated by nasogastric Coca-Cola lavage. Eur J Gastroenterol Hepatol 2002;14(7):801-803 